\title{
Substance Identification in Anti-Doping Control-Some Issues
}

\author{
Mats Larsson* \\ Department of Physics, Stockholm University, Sweden \\ *Corresponding author: Mats Larsson, Department of Physics, AlbaNova University Center, Stockholm University, Sweden. \\ To Cite This Article: Mats Larsson. Substance Identification in Anti-Doping Control-Some Issues. 2020 - 9(4). AJBSR.MS.ID.001404. DOI: 10.34297/ \\ AJBSR.2020.09.001404.
}

Received: 眥 July 02, 2020; Published: 㪢 July 08, 2020

\section{Introduction}

When an athlete is accused of an anti-doping rule violation according to the World Anti-Doping Code (WADC), usually the only evidence available to the ruling body is the presence of a prohibited substance, metabolite or marker in the athlete's sample, as determined by a laboratory accredited by the World Anti-Doping Agency (WADA). The laboratory enjoys a presumption, which means that if the analysis has been conducted according to WADA's International Standard for Laboratories and technical documents, it cannot be challenged by the athlete. It speaks for itself that given the enormous consequences for an athlete to be found guilty of an antidoping violation, and the singular importance attributed to the laboratory, the analysis procedure and the underpinning science must be impeccable. Small molecules such as anabolic steroids and their metabolites are usually detected by means of Gas Chromatography (GC) coupled to tandem Mass Spectrometry (MS/ MS) because of the selectivity and sensitivity offered by GC-MS/MS [1], and the triple quadrupole instrument is often used as platform.

The first quadrupole selects one diagnostic ion, which is then colliding with a target gas in the second quadrupole, followed by the selection of product ions by the third quadrupole. Selected reaction monitoring (also called multiple reaction monitoring) uses two diagnostic precursor-product ion transitions (qualifier ions) and one ion transition for normalization (target ion) [2]. In WADA terminology the target ion is called base peak and serves to normalize the other signals between 0 and $100 \%$. The most recent technical document regulating selected reaction monitoring requires that two ion transitions shall be monitored [3]. In an ongoing doping case, it has become obvious that the Stockholm Doping Control Laboratory has misunderstood the technical document [3] and has counted the base peak as one ion transition they monitor. In the previous technical document (WADA2010IDCR [3]), only one ion transition was allowed to be monitored under special circumstances. Using the operating procedure of the Stockholm Laboratory, this would mean comparing base peaks for the sample and the reference, an obviously meaningless exercise. It is of course disturbing that a WADA accredited laboratory has a Standard Operating Procedure not fulfilling the requirements in a WADA technical document

Sample degradation is a well-known problem when the sample cannot be promptly delivered to the laboratory [4]. Microorganisms present in the freshly voided urine will multiply rapidly and give rise to a chemical noise in the mass spectrum [5], i.e. signals arising from any unwanted species. For complex mixtures such as degraded urine sample, detection limits in mass spectrometry are usually limited by chemical noise [6]. Unfortunately, WADA does not make any distinction between chemical noise and electronic noise [3]. A signal-to-noise ratio larger than three-to-one is required [3], and it is stated that peaks clearly interfered by partially coeluting substances should be avoided. However, no attempt of quantification is made.

This is deplorable since a straight forward criterion is available in the scientific literature: chromatographic peak shape must be Gaussian [7]. Figure 1 illustrates graphically the effect of a degraded sample for which the signal is interfered by an unknown co-eluting substance. The signal-to (chemical)-noise is clearly much less than 3:1 and the shape is not Gaussian. In WADC2010IDCR [3], the analyst was permitted to use peak-resolving software. Surprisingly, this option was removed in the most recent technical document 2015IDCR [3]. 


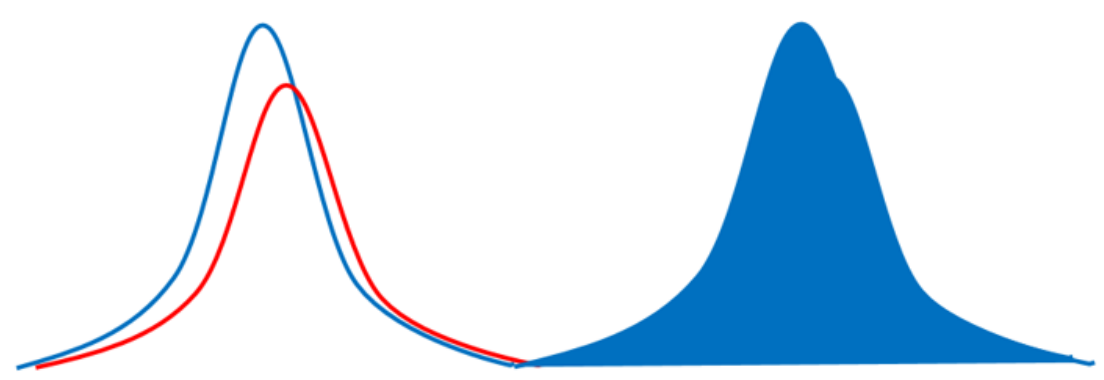

Figure 1: The blue curve is the signal, the red curve a signal from a co-eluting substance in the degraded sample. The total signal observed by the analyst is shown to the right. It clearly does not have a Gaussian shape.

\section{Acknowledgement}

The author would like to thank Richard Thomas, Stockholm University, for valuable discussions.

\section{Reference}

1. Borden SA, Palaty J, Termopoli V, Famiglini G, Cappiello A, et al. (2020) Mass spectrometry analysis of drugs of abuse: Challenges and emerging strategies. Mass Spectrom Rev p.1-42.

2. Maurer HM 2020 Pitfalls in drug testing by hyphenated low- and highresolution mass spectrometry. Drug Test Anal 12: 172-179.

3. WADA (2015) TD2015IDCR / World Anti-Doping Agency.
4. Tsivou M, Livadara D, Georgakopoulos DG, Koupparis MA, Atta Politou J, et al. (2009) Stabilization of human urine doping control samples. II. Microbial degradation of steroids. Anal Biochem 388(1): 146-154.

5. Cooks RG, Bush KL (1982) Counting molecules by desorption ionization and mass spectrometry/mass spectrometry. J Chem Educ 59: 926-933.

6. Hassell KM, LeBlanc YC, McLuckey SA (2011) Chemical noise reduction via mass spectrometry and ion/ion charge inversion: Amino acids. Anal Chem 83(9): 3252-3255.

7. Fitzgerald RL, Griffin TL, Yun YM, Godfrey RA, West R, et al. (2012) Dilute and shoot: Analysis of drugs of abuse using selected reaction monitoring for quantification and full scan product ion spectra of identification. J Anal Toxicol 36(2): 106-111. 